6

\title{
Behaviour of large scale structures of the electron content as a key parameter for range errors in GNSS applications
}

\author{
NORBERT JAKOWSKI $\left({ }^{1}\right)$, REINHART LEITINGER $\left({ }^{2}\right)$ and LUIGI CIRAOLO $\left({ }^{3}\right)$ \\ $\left(^{1}\right)$ Deutsches Zentrum für Luft und Raumfahrt (DLR), Institut für Kommunikation und Navigation (IKN), \\ Neustrelitz, Germany \\ ${ }^{2}{ }^{2}$ Institute of Physics, Institute for Geophysics, Astrophysics and Meteorology (IGAM), \\ University of Graz, Austria \\ $\left(^{3}\right)$ Istituto di Fisica Applicata «Nello Carrara» (IFAC), Firenze, Italy
}

The Total Electron Content (TEC) of the ionosphere is a key parameter for describing the ionospheric state. This paper deals with the large scale behaviour of TEC under low and high solar activity conditions. Large scale structures of the plasma density are formed by fundamental ionospheric processes mainly driven by solar radiation input, neutral winds and electric fields. The monitoring of large scale structures contributes to a comprehensive understanding of these coupling mechanisms which are rather complex particularly under perturbed geomagnetic conditions. The paper addresses techniques to monitor TEC with sufficient accuracy of a few TEC units $\left(10^{16} \mathrm{~m}^{-2}\right)$ to measure large scale structures over Europe and over the polar areas. The availability of GPS data from global GPS receiver networks as e.g., those from the International GPS Service (IGS) is dense enough to generate TEC maps on a continuous base. A model assisted technique is briefly described for mapping TEC over the European and polar areas. A statistical estimation of horizontal TEC gradients reveals large scale gradients of up to about 6 TECU/1000 km under high solar activity conditions at an occurrence probability level of about $1 \%$. Occasionally, during severe ionospheric storms this value may increase by a factor of 10 or even more. A close correlation of large scale gradients and the geomagnetic activity has been found giving the chance to forecast TEC gradient amplitudes by using predicted geomagnetic indices. Since TEC is proportional to first-order range errors in Global Satellite Navigation Systems (GNSS) such as the US GPS and the Russian GLONASS the study of the behaviour of this parameter has a practical meaning in GNSS based navigation and positioning. The paper addresses the close relationship between TEC and ranging errors in GNSS. Having in view Galileo, the planned Europe's own global satellite navigation system, some aspects related to the mitigation of ionospheric propagation errors within the European Geostationary Navigation Overlay System (EGNOS) are discussed. Since EGNOS will augment the two above mentioned satellite navigation systems and make them suitable for safety critical applications such as flying aircraft or navigating ships through narrow channels the ionospheric propagation errors have to be mitigated as much as possible.

\subsection{INTRODUCTION}

Electromagnetic radio waves transmitted from satellites of Global Navigation Satellite Systems (GNSS) such as GPS or GLONASS interact with the ionospheric plasma. 
In Global Navigation Satellite Systems using $L$-band frequencies, the ionosphere still causes signal delays that correspond with range errors of up to about $60 \mathrm{~m}$. In a first order approximation the range error is proportional to the integral of the electron density along the ray path (Total Electron Content, TEC) and can be mitigated by a simple linear combination of $L 1$ and $L 2$ phases due to the dispersive nature of the ionosphere.

However, horizontal gradients in the ionospheric ionisation and ionospheric irregularities producing phase fluctuations and radio scintillations may also cause a significant degradation of navigation signals up to loss of lock. Strong TEC gradients, Traveling Ionospheric Disturbances (TIDs) and phase fluctuations are often correlated with the development of geomagnetic/ionospheric storms.

Therefore, a comprehensive knowledge of the ionosphere is required for studying the impact of the ionospheric plasma on GNSS applications. Fortunately, the GNSS technique itself provides a unique opportunity for high resolution monitoring the ionosphere on global scale as discussed in Section 6.2. Although the GNSS technology is well-suited for monitoring ionospheric processes at quite different temporal and spatial scale, in this study we focus on large scale ionospheric processes and their impact on GNSS signals (e.g., Jakowski et al., 2001).

The information about the ionospheric ionisation is derived from dual frequency code and carrier phase measurements at $L$-band frequencies transmitted permanently by the global navigation satellite systems GPS and GLONASS.

\subsection{GROUND BASED TEC MONITORING}

To derive the Total Electron Content (TEC) of the ionosphere from ground based GPS measurements various techniques have been developed (e.g., Wilson and Mannucci, 1993; Ciraolo et al., 1994; Sardón et al., 1994)

Generally speaking, the procedure can be derived in several steps:

1) Computation of the differential delays of code and carrier phases along all available GPS receiver radio links. In the first-order-approximation the differential phases are proportional to the integral of the electron density along the ray path (TEC). The corresponding GPS data files, usually provided in a Receiver Independent Exchange (RINEX) format, can be downloaded from archives of the International GPS Service (IGS) or other data centers offering this type of data.

2) Computation of biased TEC values for each signal path. Whereas the noisy travel time measurements by the code phases provide an absolute measure of TEC affected by a quasi-constant bias, the higher precise differential phase delays yield only a relative measure of TEC. For saving the high accuracy of the differential phases, it is a common practice to level them into the code phases by the least squares method ( $c f$. fig. 6.1).

3) Calibration of the biased TEC values. Strictly speaking, there is one bias for each satellite and for each station, which can be assumed to be constant over long periods of time. The evaluation of these biases constitutes the process of calibration, after which one will obtain evaluations of actual slants (but still affected by errors). Usually the calibration requires a simplified ionospheric model e.g., by a polynomial approach. Naturally, the chosen approaches are different by different research groups (e.g., Wilson and Mannucci, 1993; Ciraolo et al., 1994; Sardón et al., 1994).

The TEC data discussed in this paper are calibrated by a Kálmán filter technique described by Sardón et al. (1994).

4) Mapping the calibrated TEC to the vertical at the pierce point of the ray path with the single layer ionospheric shell at a fixed height between 300 and $450 \mathrm{~km}$. The corresponding mapping function is also used to compute the slant ionospheric propagation error if slant TEC information is provided.

5) Construction of TEC maps from individual TEC measurements distributed over a certain region or on global scale. The generation of TEC maps requires the availability of a sufficient number of 


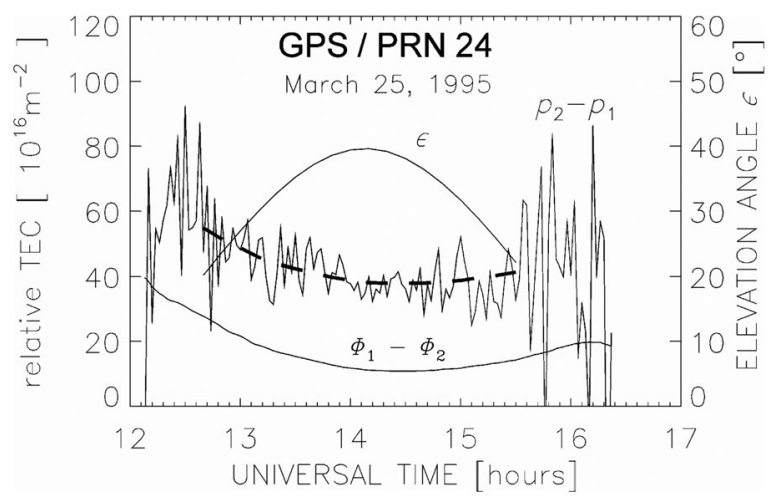

Fig. 6.1. Differential code (P2-P1) and carrier $(\phi 1-\phi 2)$ phases of GPS signals (PRN 24) measured in Neustrelitz on 25 March 1995. The dashed line indicates the least squares levelled differential carrier phases used for further computation.

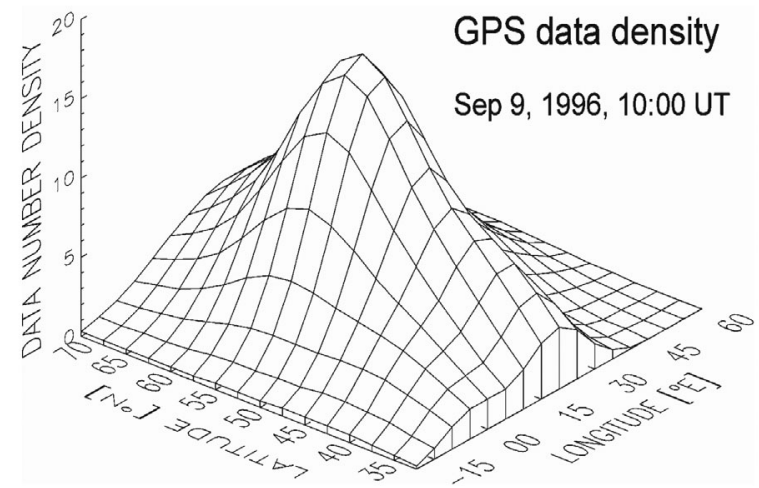

Fig. 6.2. Data coverage indicated by the sum of weighting functions of measuremens at each grid point of the corresponding TEC map for 9 September 1996, 10:00 UT.

measurements ( $c f$. fig. 6.2). As already noticed earlier, different approaches are applied by different research groups (e.g., Jakowski, 1996; Hernández-Pajares et al., 1997, 1999).

Because the large scale behaviour of TEC discussed in the following sections is based on the analysis of regional TEC maps routinely generated in DLR Neustrelitz since 1995 (http://kn.nz.dlr.de/) the corresponding procedure is described here in more detail.

After the slant TEC values are transformed to the vertical TEC at the pierce points with the single layer ionosphere shell at $400 \mathrm{~km}$ height, the maps are constructed by a data assimilation technique.

To ensure a high reliability of the TEC maps also in case of only a few measurements or at greater distances from the measuring points, the measured data are combined with the empirical TEC model NTCM2 (e.g., Jakowski 1996, 1998). To obtain reliable TEC values at the grid points, a weighting process between nearest measured and model values is carried out for each grid point value (grid 

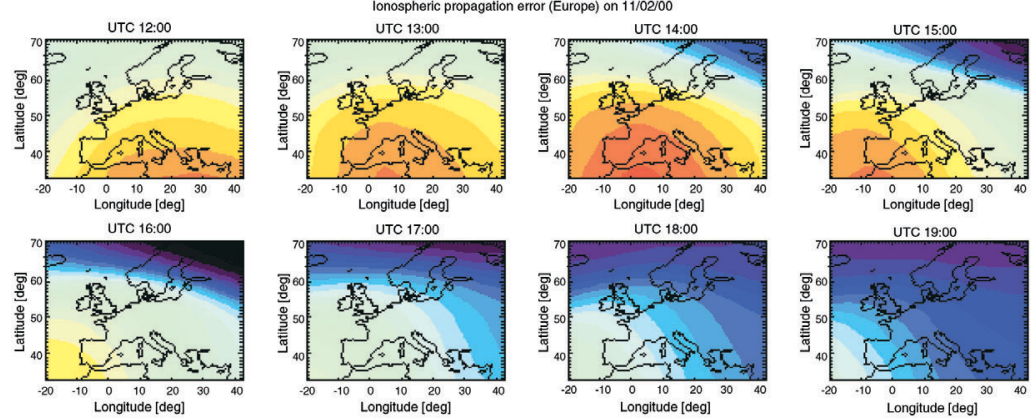

UTC 17:00

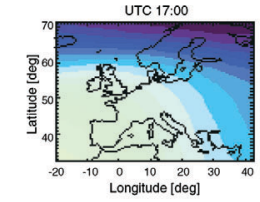

UTC 18:00

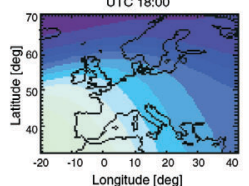

UTC 19:00
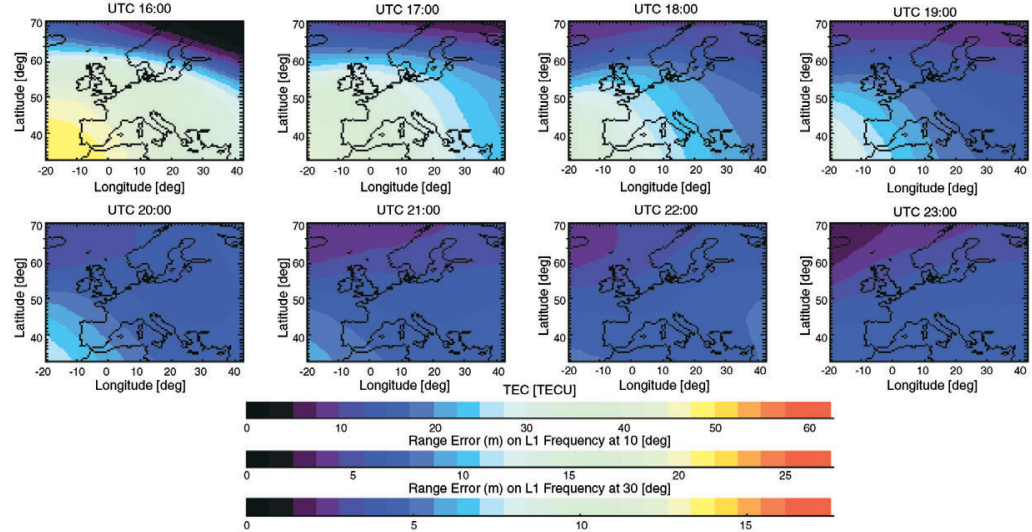

Fig. 6.3. Hourly maps of vertical TEC and corresponding range errors over Europe on 11.02.2000 derived from GPS ground station measurements of IGS.

spacing is $2.5^{\circ} / 5^{\circ}$ in latitude/longitude). Since the IGS network provides about 60-200 TEC data points over the European region considered, the generated TEC maps are suited to monitor large scale horizontal ionisation structures. It has to be taken into account that the data are usually uneven distributed over Europe as is shown for a selected snapshot in fig. 6.2. Nevertheless, due to the construction technique grid points at the margin area are also influenced by the overall measurements.

The absolute accuracy of the gridded TEC values has been estimated to lie in the order of a few $10^{16}$ electrons per square meter (Jakowski and Sardón, 1996). This accuracy is high enough to monitor large scale perturbation processes due to severe space weather effects. COST 271 has initiated the establishment of an TEC Evaluation DAta bank - TECEDA at DLR/IKN Neustrelitz to enhance capabilities of data comparison and comprehensive ionospheric studies (cf. Stamper et al., 2004). A comparison of vertical TEC data, derived over the ionosonde station Athens from the regularly processed TEC maps, with estimated ionospheric TEC data (ITEC), derived from electron density profiles of the digisonde, shows a rather good agreement. As it has been demonstrated by Belehaki et al. (2003), the difference has even the potential to get information about the plasmaspheric electron content. To enable comprehensive studies of ionospheric processes and ionospheric propagation effects on GNSS, an international HIgh RAte GPS/GLONASS measuring Campaign (HIRAC) was initiated together with the International GPS Service (Feltens et al., 2001).

After the HIRAC measuring campaign was carried out in April 2001 including more than 100 stations on global scale, a huge amount of data sampled once per second are available now for further analysis. First analysis samples revealed interesting effects such as TIDs and enhanced differential carrier phase irregularities under disturbed conditions (Kersley et al., 2004).Typical TEC maps for the European area in the range $20^{\circ} \mathrm{W}<\lambda<40^{\circ} \mathrm{E} ; 32.5^{\circ} \mathrm{N}<\phi<70^{\circ}$ are shown in fig. 6.3 where the corresponding range errors at selected elevation angles are also plotted at the lower panel (http://www.kn.nz.dlr.de/daily/tec-eu). 
In order to monitor large scale processes in the more intensively disturbed high latitude ionosphere, the same mapping technique as described for the European area is applied for geographic latitudes $\phi>50^{\circ} \mathrm{N}$ and $\phi>50^{\circ} \mathrm{S}$. To do this in an adequate manner, a preliminary model for the polar TEC (NTCMP-1) was established. Thus, in the same way as described above, polar maps of vertical TEC were derived for a grid consisting of 768 points within the latitude ranges $50^{\circ} \mathrm{N} \leq \phi \leq 90^{\circ} \mathrm{N}$ and $50^{\circ} \mathrm{S} \leq \phi \leq 90^{\circ} \mathrm{S}$ during the COST 271 action period. The corresponding actual northern polar TEC maps are currently available in the Web via http://www.kn.nz.dlr.de/daily/tec-np/.

\subsection{Regular behaviour of TEC}

It is evident that ionosphere induced propagation errors are easier to handle if signals travel through an unperturbed ionosphere with low temporal and spatial variability. Nevertheless, the ranging error cannot be ignored as pointed out in more detail in Section 6.5. Since the unperturbed ionosphere fits quite well with smooth structures that are well represented by monthly averages or medians, the corresponding range errors can be estimated by ionospheric models. Thus, single frequency GPS customers can apply a model whose 8 coefficients are daily upgraded and permanently broadcasted to the GPS receiver. This GPS model, developed by Klobuchar (1987) - therefore often called Klobuchar-model - is able to remove range errors up to about $50 \%$. Three dimensional ionospheric models such as IRI or Nequick (Hochegger et al., 2000) are suited to integrate along the real ray path of GNNS signals through the ionosphere thus enabling the study of higher order propagation error effects.

Figure 6.4 shows a comparison of measured TEC with the three dimensional IRI model IRI-95 and the two dimensional TEC model NTCM2. The latter is used as a background model for the generation of TEC maps (see Section 6.2.). As expected, the figure shows clearly that the TEC structures are smooth both for the monthly median TEC values as well as for the model data. The latitudinal TEC gradients are comparable for all data sets.
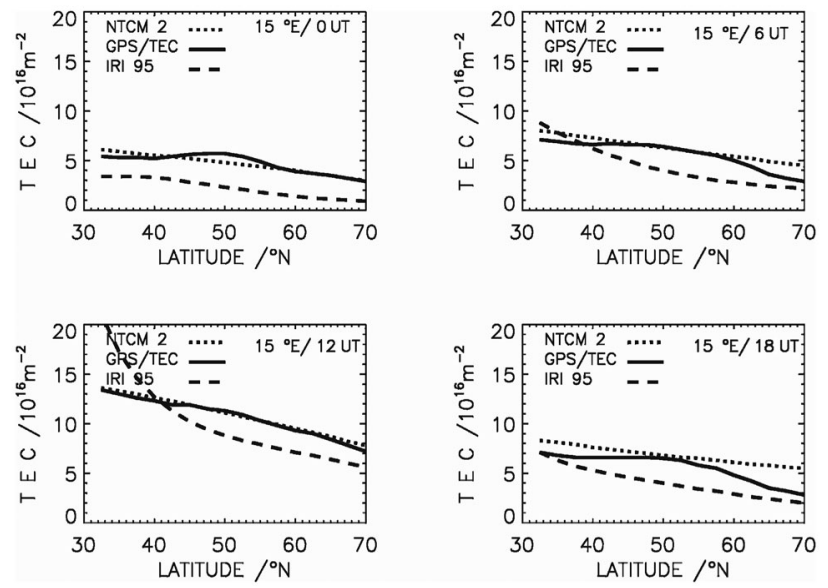

Fig. 6.4. Monthly median TEC monitoring data along $15^{\circ} \mathrm{E}$ for October 1996 in comparison with model values from IRI-95 and the Neustrelitz model NTCM2. 


\subsection{LARGE SCALE STRUCTURES OF TEC}

Because the ionospheric ionisation is subjected to a certain natural variability of about $30 \%$, instantaneous TEC snapshots show a more structured ionisation over Europe if compared with monthly medians as shown in fig. 6.4. To get a quantitative impression, the distribution of the strength of latitudinal and longitudinal TEC gradients measured in $10^{15} \mathrm{~m}^{-2} / 100 \mathrm{~km}($ TECU/1000 km) is shown in fig. 6.5 for the low and high solar activity years 1995-1996 and 1999, respectively.

The latitudinal and longitudinal gradients of TEC were derived from vertical TEC maps whose generation is described in Section 6.2. Due to the smoothing effect of the applied model assisted mapping technique (half width of the weighting function around a single measurement was about $500 \mathrm{~km}$ ) and the limited grid point resolution small scale structures are not reflected in the estimations shown in fig. 6.5. Hence, the TEC maps and the corresponding statistics ignoring diurnal and seasonal dependences describe large scale effects with scale length $\geq 500 \mathrm{~km}$. Principally, the gradients over Europe grew to higher values under High Solar Activity (HSA) conditions in 1999 compared with those deduced for the Low Solar Activity (LSA) years 1995-1996. Furthermore, it can be seen that latitudinal and longitudinal gradients have a similar width in the order of a few TECU/1000 km. Whereas the longitudinal gradients are nearly symmetric around zero, the latitudinal gradients are shifted to negative values ( -1 TECU/1000 km for LSA and -2 TECU for HSA) thus indicating the well-known increase in TEC towards the equator due to the solar zenith angle control of the ionisation. This conclusion is consistent with the observation of stronger gradients at $35^{\circ} \mathrm{N}$ than at $65^{\circ} \mathrm{N}$. Under HSA conditions in 1999 a gradient of 0.5 TECU/100 km is observed with a probability of about $3.5 \%$ for east-west and $4.5 \%$ for north-south directions.

It is obvious that the highest gradients, exceeding the value of $0.5 \mathrm{TECU} / 100 \mathrm{~km}$ considerably, are expected to occur in the course of ionospheric storms which cause highly dynamic temporal and spatial changes of the ionospheric plasma ( $c f$. fig. 6.8). Whereas rapid small scale irregularities may cause radio scintillations, large scale effects characterized by a slower temporal variability of TEC, may cause ranging problems in differential GNSS networks such as the Wide Area Augmentation Systems (WAAS) or the European Geostationary Navigation Overlay System (EGNOS).

To characterize storm induced gradient enhancements, differential TEC maps, e.g., percentage differences from the median behaviour according to $\triangle \mathrm{TEC}=100 *(\mathrm{TEC}-\mathrm{TECmed}) / \mathrm{TEC}$ ed are helpful to draw conclusions concerning the driving forces of ionospheric storm processes such as electric fields or perturbation induced wind (e.g., Jakowski et al., 1999a,b). Furthermore, statistical studies showed a characteristic storm pattern of percentage TEC deviations from monthly medians for winter and summer storm (e.g., Foerster and Jakowski, 2000).

In this section the selected case studies focus on large scale plasma signatures usually driven by significant space weather events.

The geomagnetic storm on 6 April 2000 is characterized by a strong geomagnetic activity (see $K p$ in fig. 6.6) and an intensive and long lasting auroral hemisphere power index (Foster et al., 1986).

After the shock wave has been detected by particle detectors onboard NASA's Advanced Composition Explorer spacecraft (ACE) around 16:00 UT, various space weather effects were detected at different spheres down to the Earth surface such as polar lights (Jakowski et al., 2002b).

The corresponding TEC data have been derived from the routinely produced TEC maps over the North pole as previously discussed in Section 6.2.

The highly variable temporal and spatial structures are probably due to particle precipitation through the cusp region and strong plasma convection across the pole due to perturbation electric fields. We assume that the strong impact on the magnetosphere/ionosphere systems in the evening hours of 6 April is due to the southward direction of the interplanetary magnetic field $(B z<0)$ that has been measured from about 17:00 until 24:00 UT (Jakowski et al., 2002b). Principally, the polar ionisation is strongly irregular between 17:00 and 24:00 UT. The temporal variation indicates a strong irregular structure starting at about 17:00 UT at $\lambda=280^{\circ} \mathrm{E}$ which is close to the magnetic zero lon- 


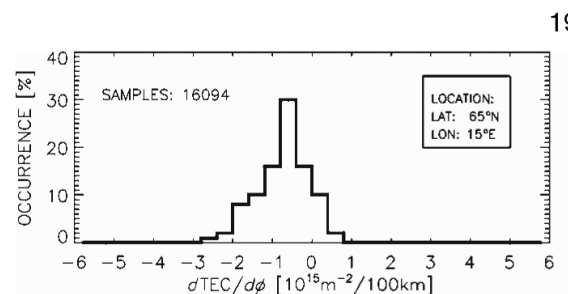

1995-1996
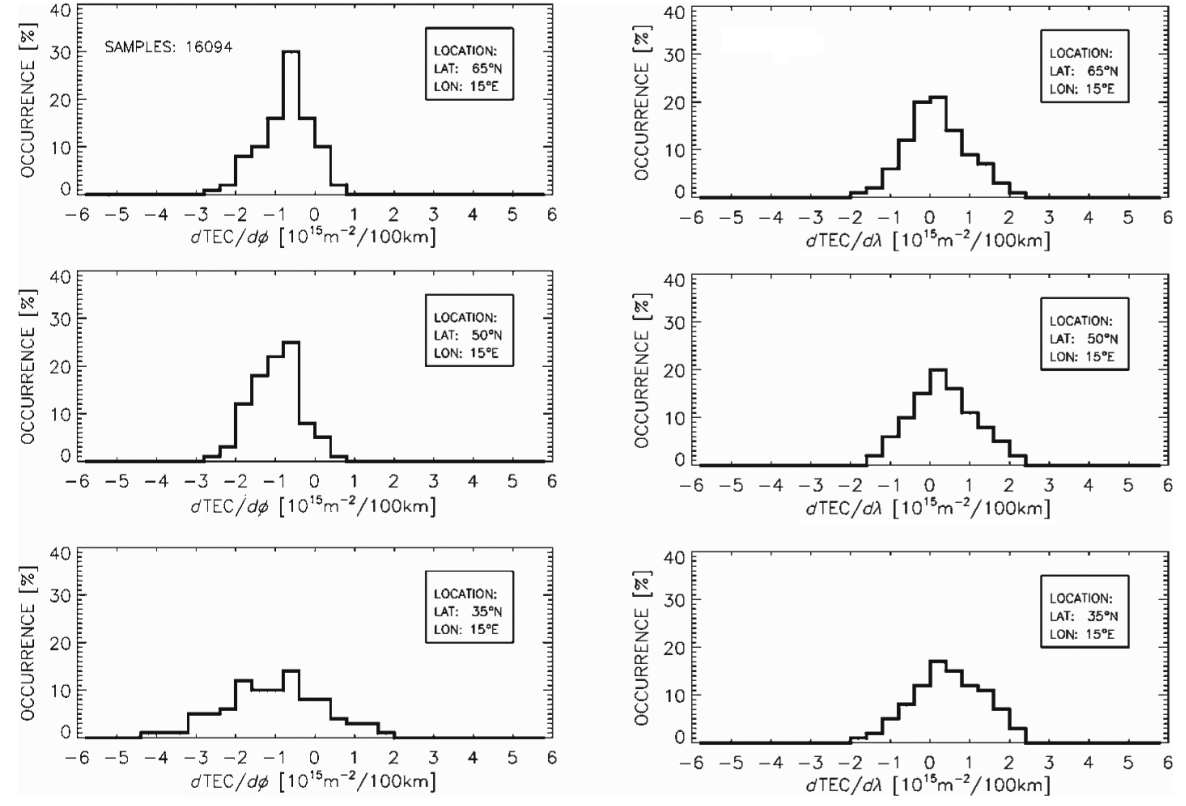

1999
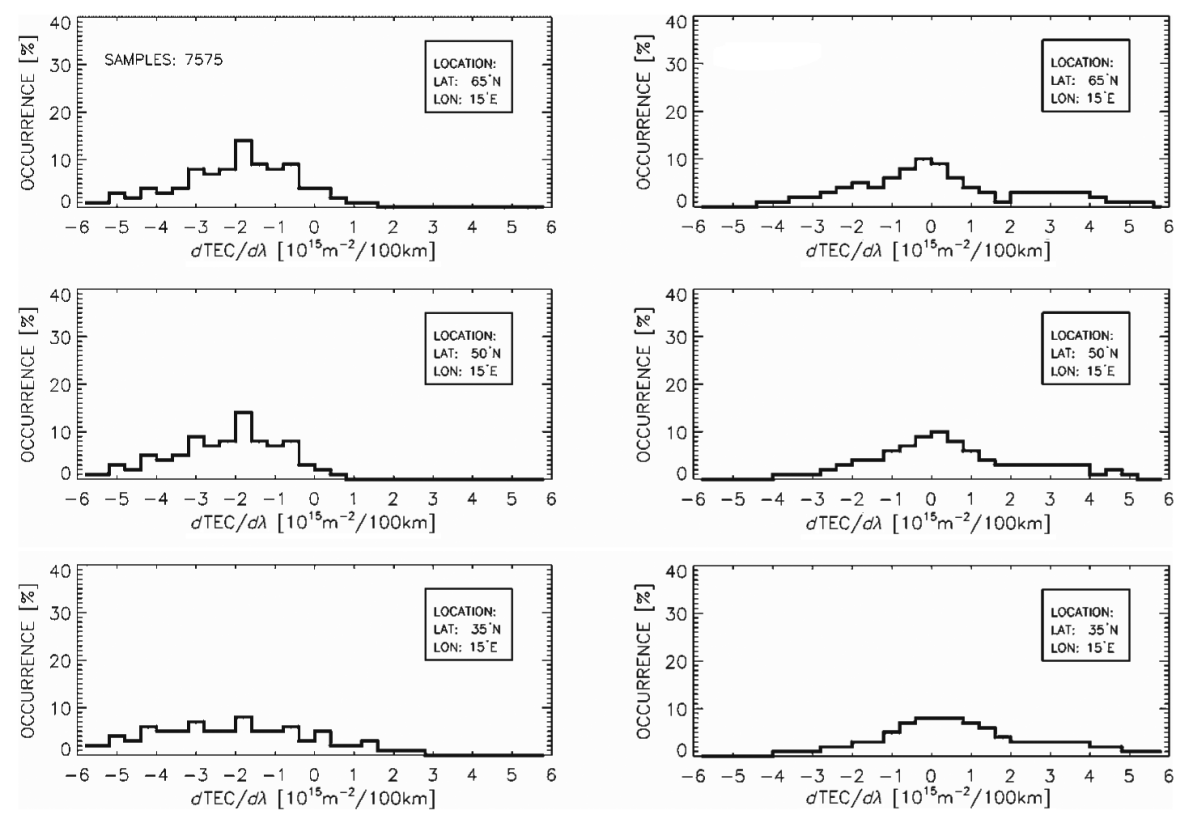

Fig. 6.5. Percentage distribution of horizontal TEC gradients for low solar activity (upper panel: 1995-1996) and high solar activity conditions (lower panel: 1999). 


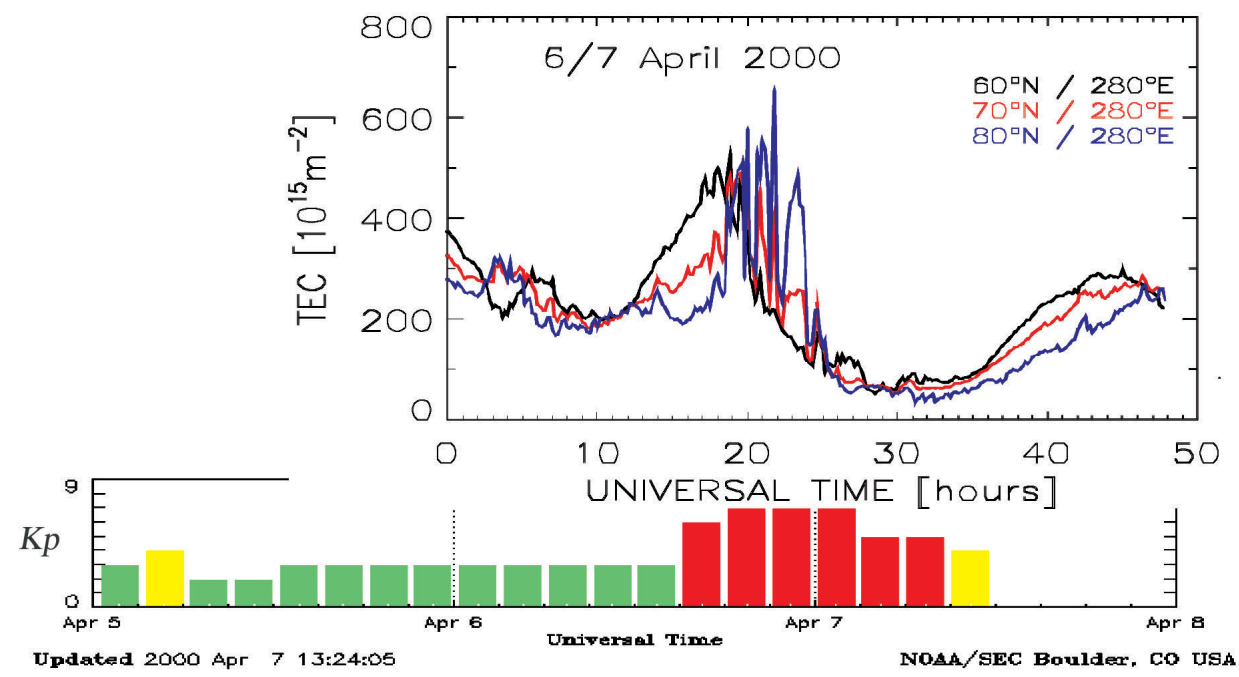

Fig. 6.6. $K p$ data published by NOAA/SEC, Boulder in comparison with TEC data derived from polar TEC maps at different latitudes along the $280^{\circ} \mathrm{E}$ meridian.

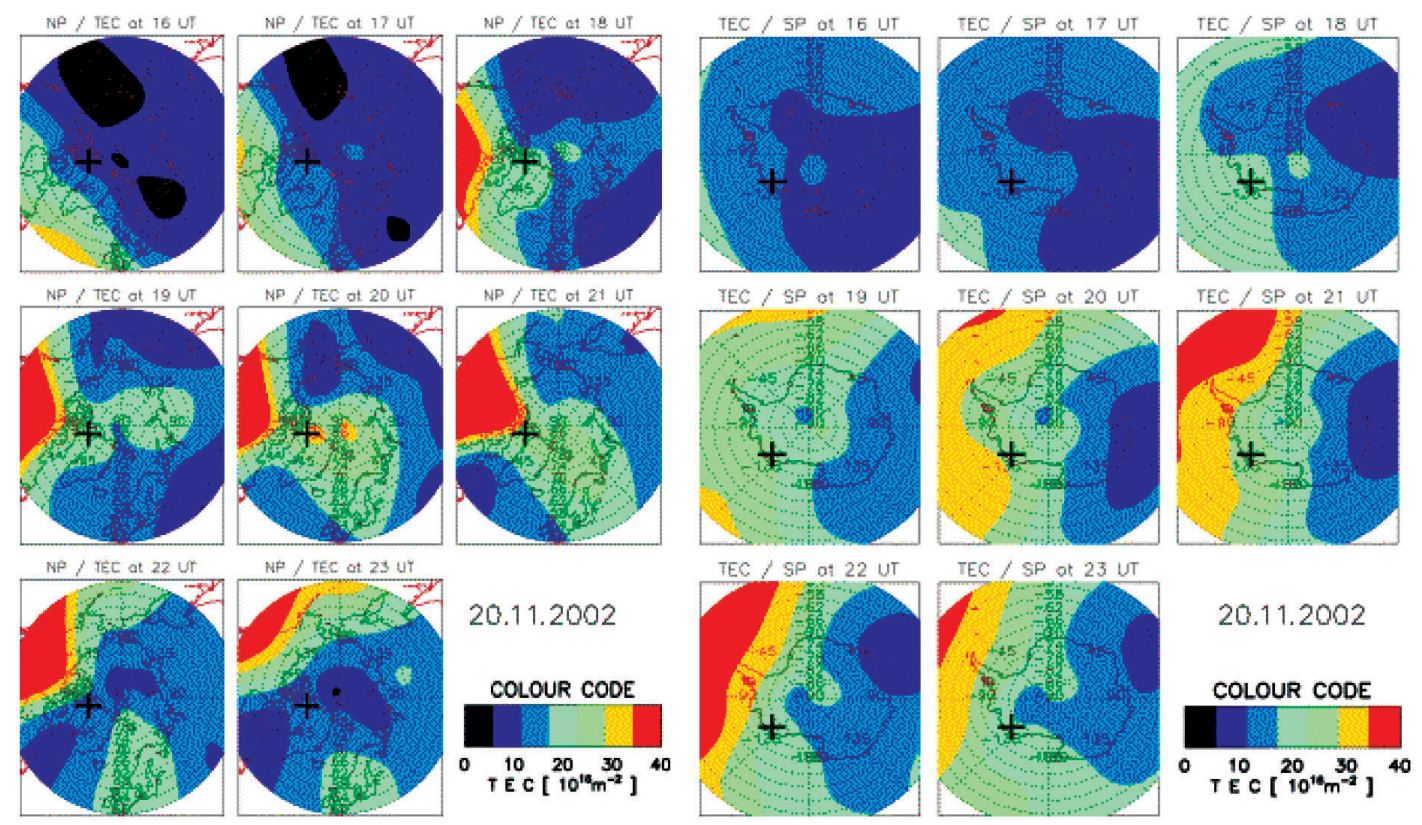

Fig. 6.7. Comparison of hourly TEC maps over the North (left panel) and South (right panel) pole areas during the geomagnetic storm on 20 November 2002. 
gitude. The amplitude of TEC can reach extremely high values of up to $60 \times 10^{16} \mathrm{~m}^{-2}(60 \mathrm{TECU})$ that has a severe impact on accuracy and reliability of GNSS navigation. Strong phase fluctuations were observed at mid-latitude European GPS stations in particular around 23 UT.

It is interesting that the measurements at the Italian station in Ispra were practically not perturbed by this storm. This observation indicates the existence of significant horizontal structures producing strong TEC irregularities and in conjunction with this also severe phase fluctuations.

To understand the complexity of large scale ionospheric perturbations, correlative studies between north and south pole ionospheric features should be meaningful. Although the number of Antarctic GPS ground stations is still strongly limited, preliminary studies are already possible as shown in fig. 6.7. Both polar TEC maps illustrate strong and permanent changing gradients of the ionospheric ionisation induced and controlled by dynamic forces in the course of the space weather event on 20 November 2002. Due to the strong magnetosphere-ionosphere coupling at high latitudes the polar regions are very sensitive to space weather events. To characterize the perturbed ionosphere in an early stage for warning GNSS customers, it is proposed to characterize the perturbation degree of the ionosphere by an ionospheric perturbation index as pointed out recently (Jakowski et al., 1999b; Jakowski, 2003).

The complex perturbation processes at high latitudes produce strong gradients in the three dimensional plasma structure characterized by quite different scale lengths. Whereas effects due to particle precipitation are mainly characterized by a small scale patchy structure, plasma convection due to the action of the convection electric field of magnetospheric origin have large scale characteristics visible in the TEC maps. Such a well developed plasma convection process propagating from the day-side towards the night-side can be seen in fig. 6.7 in the TEC maps from 19:00-21:00 UT. Consequently, the occurrence of perturbation induced TEC gradients should be well correlated with geomagnetic indices such as $K p$ or $A p$. To check this hypothesis, latitudinal TEC gradients derived from a few selected grid points of the European TEC maps were cross correlated with the three-hourly Ap index.

Figure 6.9 indicates clearly that horizontal TEC gradients are closely correlated with the geomagnetic activity, here represented by $A p$. In agreement with the occurrence distribution of horizontal TEC gradients shown in fig. 6.5, the large scale gradients increase with the level of ionisation measured by
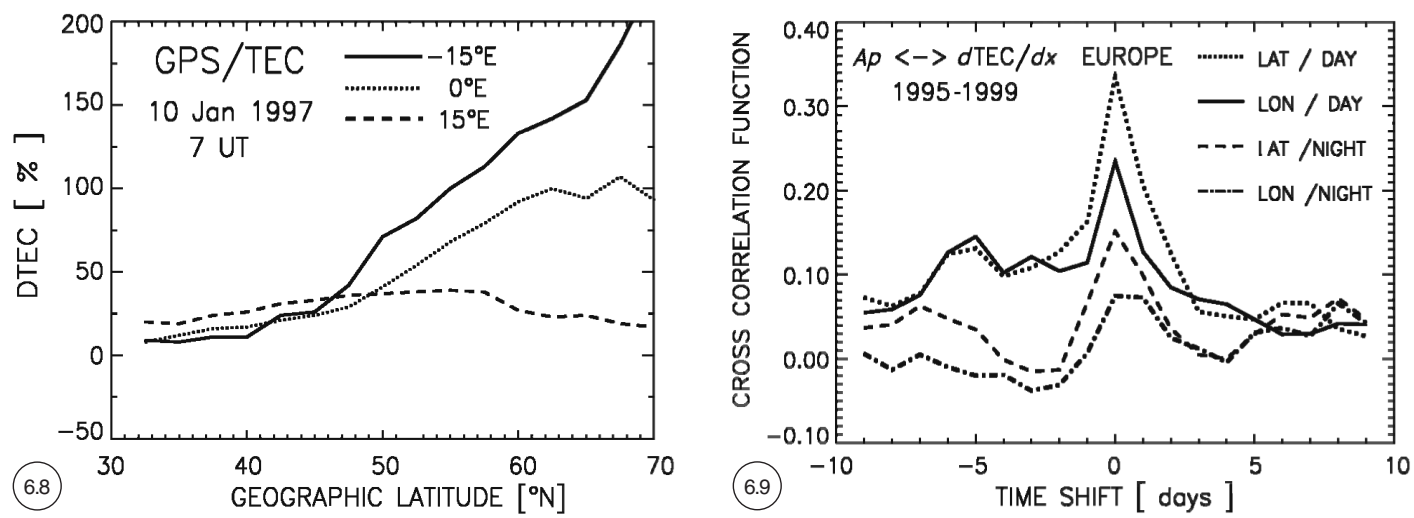

Fig. 6.8. Strong latitudinal gradients in the percentage storm pattern during the geomagnetic storm on 10 January 1997 indicating also strong longitudinal differences at latitudes $>50^{\circ} \mathrm{N}$.

Fig. 6.9. Cross-correlation between latitudinal and longitudinal TEC gradients and the three-hourly Ap index for data sets taken from European TEC maps that cover the years 1995-1999. 

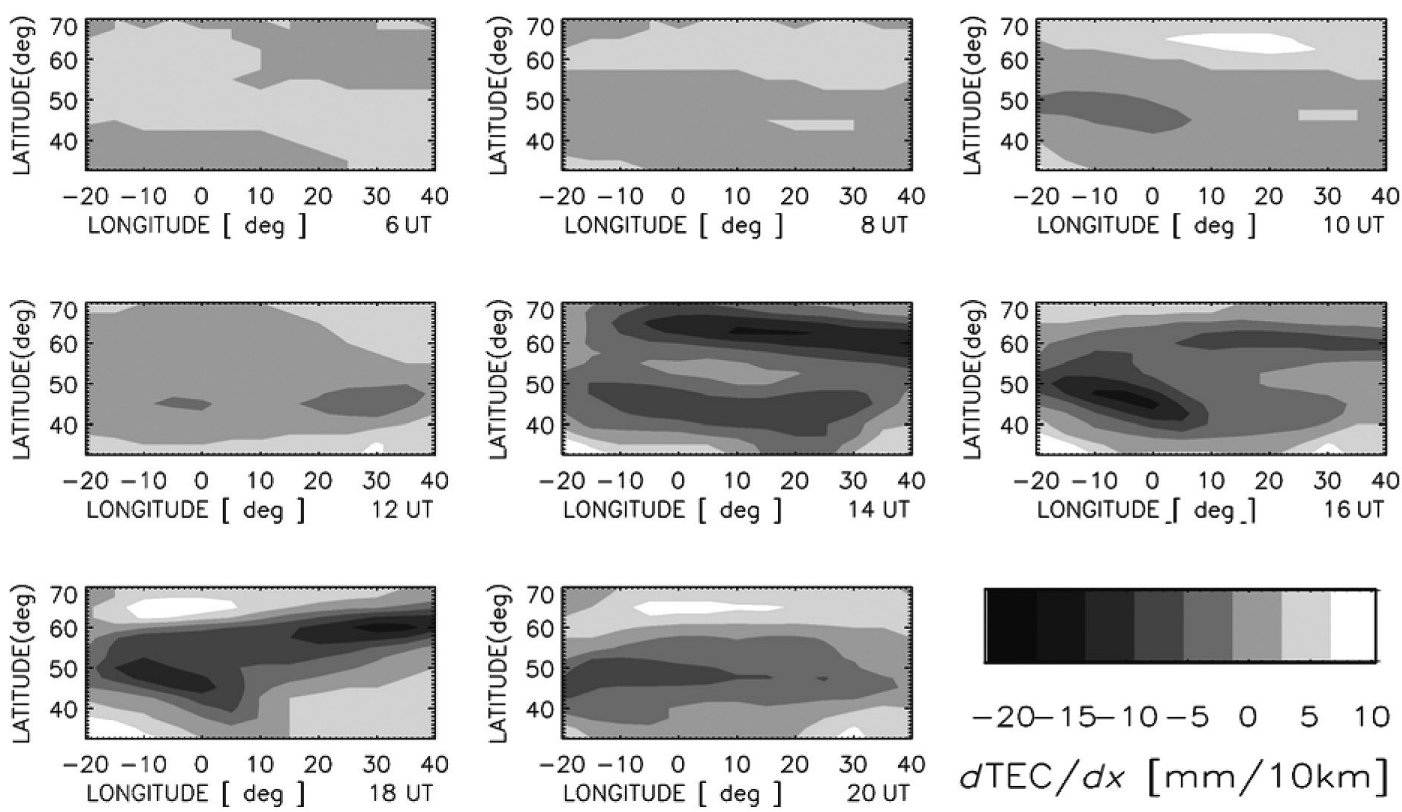

Fig. 6.10. Two-hourly plots of TEC gradients in the course of the geomagnetic storm on 26 March 1995.

TEC, i.e. the highest gradients are observed at day-time. Furthermore, it is evident from fig. 6.9 that perturbation induced gradients are stronger in the latitudinal than in the longitudinal direction.

Strong gradients in ionisation are often accompanied by enhanced carrier phase fluctuations (e.g., Jakowski et al., 2002a). Consequently, accuracy and reliability of differential GNSS services are sensitive to the geomagnetic activity. To demonstrate how such gradient structures may develop and may be distributed over Europe, fig. 6.10 shows two-hourly snapshots of TEC gradients during the storm on 26 March 1995.

Assuming a distance of $50 \mathrm{~km}$ between a customer and the nearest reference station, fig. $6.10 \mathrm{in}$ dicates a de-correlation of more than $10 \mathrm{~cm}$, that will cause difficulties in solving the carrier phase ambiguities in the precise positioning algorithms. Gradient maps of this type will be generated in the near future as a data product within a specific space weather service for precise navigation applications in DLR Neustrelitz (http://www.kn.nz.dlr.de/swippa/). In this context it should be mentioned that Differential GPS (DGPS) networks reported severe difficulties to maintain their service during the recent large geomagnetic/ionospheric storm on 30 October 2003 (e.g., Jakowski et al., 2003).

\subsection{RANGE ERROR CORRECTIONS}

As already mentioned in the previous sections, the ionospheric propagation error is proportional to the TEC along the ray path in the first order approximation. Excluding the impact of small scale irregularities the propagation effects stem from the difference between free space signal propagation and propagation through the ionised and neutral atmosphere in the geometric optics domain (Leitinger and Putz, 1988; Leitinger, 1990, 1991). 
For example, satellite based navigation and positioning rely on «range», meaning the distance between transmitter and receiver (more accurately expressed: between well defined markers within the transmitting and receiving antenna systems, $T$ and $R$ ).

Let «true range» be $\rho=\int^{R} d s_{o}$ where $d s_{o}$ is the element of the straight line from $T$ to $R$, and compare it with the measured quantity, $S=\int_{\tau}^{k} n d s, n$ being the refractive index, $d s$ the ray path element.

The considered range error $s=\rho-S$ can be expressed as

$$
s=\left(\int_{T}^{R} d s_{o}-\int_{T}^{R} n d s_{o}\right)+\left(\int_{T}^{R} n d s_{o}-\int_{T}^{s} n d s\right)=s_{1}+s_{2} .
$$

Here $s_{1}$ means the phase difference along the line of sight and $s_{2}$ provides the excess path due to ray path bending.

In this paper we ignore the neutral gas contribution to the range error which may amount to a few meters. The radio wave propagation in the ionospheric plasma is described by the complex AppletonLassen formula which may be simplified at signal frequencies $f>>100 \mathrm{MHz}$ by the «quasi-longitudinal approximation» giving for the square of the plasma refractive index $n_{i}$

$$
n_{i}^{2} \approx 1-\frac{f_{p}^{2}}{f^{2} \pm f f_{g}|\cos \Theta|}=1-\frac{A N_{e}}{f^{2} \pm f f_{g}|\cos \Theta|}
$$

where $f_{p}$ is the electron plasma frequency, $f_{g}$ the electron gyro frequency, $\Theta$ the propagation angle (angle between ray path and geomagnetic induction vector).

Using SI units the value of $A=80.6$ can be inserted.

A series expansion for $n_{i}$ leads to an expansion of $s_{1}$

$$
s_{1} \approx \frac{1}{f^{2}} \frac{A}{2} \int_{T}^{R} N_{e} d s_{o}+\text { higher.order.terms }
$$

where $N_{e}$ represents the electron density. Considering $L$-band frequencies $f>1 \mathrm{GHz}$ as used in operational GNSS such as GPS and GLONASS, the ray bending error $s_{2}$ lies in the millimetre to centimetre range for ground based measurements because it is of order 2 in $\left(1 / f^{2}\right)$. Ignoring also the small higher order terms in $s$, the first-order range error is proportional to the total electron content

$$
\mathrm{TEC}=\int_{T}^{R} N_{e} d s_{o}
$$

along the line of sight (slant TEC). A very high value for this quantity, $400 \times 10^{16} \mathrm{~m}^{-2}$ and a signal frequency of $1.575 \mathrm{GHz}$ (GPS frequency L1) gives a first-order ionospheric range error of $65 \mathrm{~m}$. With this signal frequency the higher order ionospheric range errors are in the order of centimetres and therefore negligible in navigation applications but not necessarily in precision positioning.

The strong dispersion of a plasma compensates for the first order ionospheric range error by means of a second radio signal transmitted phase coherently with an other signal frequency. It should be noted that compensation of the first order range error overcompensates (enhances) the higher order range errors.

Radio link related range errors are not directly related to position errors. With GPS or any other GNSS the position of a receiver can be determined by means of the ranges to three satellites if their orbital positions are known (the receiver is at the apex of a pyramid the basis of which is given by 
the three satellites). The so-called «clock errors» are removed by using the range to a forth satellite.

It makes sense to distinguish between horizontal and vertical position errors. In the average the vertical ones are about twice as large as the horizontal ones. It should be noted that with unfavourable geometries the vertical position error can be larger than the largest of the range errors.

Clock error compensation also removes some common term of the range errors, according to the geometry of observations.

For various reasons Satellite Based Navigation Systems for civilian applications use single frequency receivers onboard the vehicles to be navigated. For medium precision navigation the plasma range errors are estimated from a (data driven) model. High precision navigation approaches use augmentation systems which are based on electron content observations by means of stationary dual frequency receivers. Essentially, we can distinguish three types of augmentation systems: 1) local differential GPS, 2) systems based on regional dense networks of dual frequency receivers, and 3) wide area augmentation systems.

Currently, the latter category consists of two partly operational systems, the Wide Area Augmentation System (WAAS) for the «contiguous U.S.A.» and the European Geostationary Navigation Overlay System (EGNOS) for Western and Central Europe. Both WAAS and EGNOS rely on grids of dual frequency receivers which provide the data for an electron content model which relies on data only and is fabricated by a central processing unit with an update rate and a latency of $5 \mathrm{~min}$. The model is distributed to users via geostationary satellites. The model is a regular grid of vertical electron content values (fig. 6.11). From this grid an onboard computer calculates the slant range errors for the links from the users to the visible GPS satellites. Both WAAS and EGNOS use conversion from vertical to slant by means of a projection in the penetration points of the satellite to user rays with the $350 \mathrm{~km}$ height shell («pierce point» concept).

It is essential for the user to obtain information on residual ionospheric range errors and on the availability of the system. The accuracy of these estimations depend on horizontal TEC gradients as described in the previous sections in a twofold manner: 1) the conversion from slant into vertical TEC for producing error maps and vice versa to deduce specific ray path related corrections from given maps; 2) the interpolation between the grid points which may be separated by more than $600 \mathrm{~km}$ (fig. 6.11).

As pointed out by Tsedilina et al. (1994) horizontal gradients in the ionosphere strongly affect the signal delay. Thus, developed models and algorithms for precise GPS-aircraft navigation have to be refined to reduce errors caused by spatial de-correlation of the ionosphere (Harris et al., 2001).

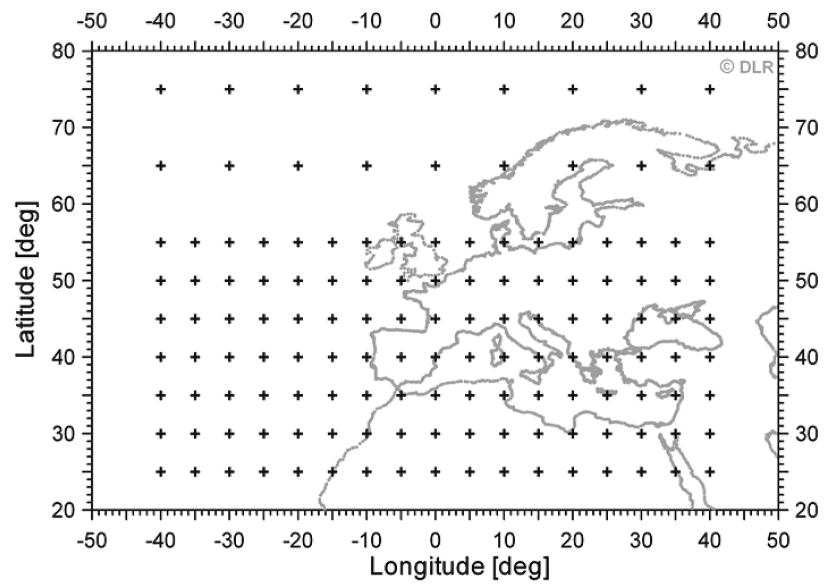

Fig. 6.11. Ionospheric grid points used for EGNOS. 
Nevertheless, there is no doubt that in high and mid latitudes the pierce point concept is acceptable. However, the system will ignore most of the smaller structures, namely those with horizontal angular scales $<<1^{\circ}$ or scale lengths $<<100 \mathrm{~km}$ and partially smoothes out intermediate scales. The smaller structures do not challenge the systems: the typical relative amplitudes of the ever present smaller scale Travelling Ionospheric Disturbances are rarely $>3 \%$. There remain the structures with angular scales between about $5^{\circ}$ and about $20^{\circ}$ which usually are considered to produce «strong horizontal gradients». Both EGNOS and WAAS rely on redundancy to detect the effect of these structures on the residual errors. The relevant algorithms (which are not revealed but considered to be in-
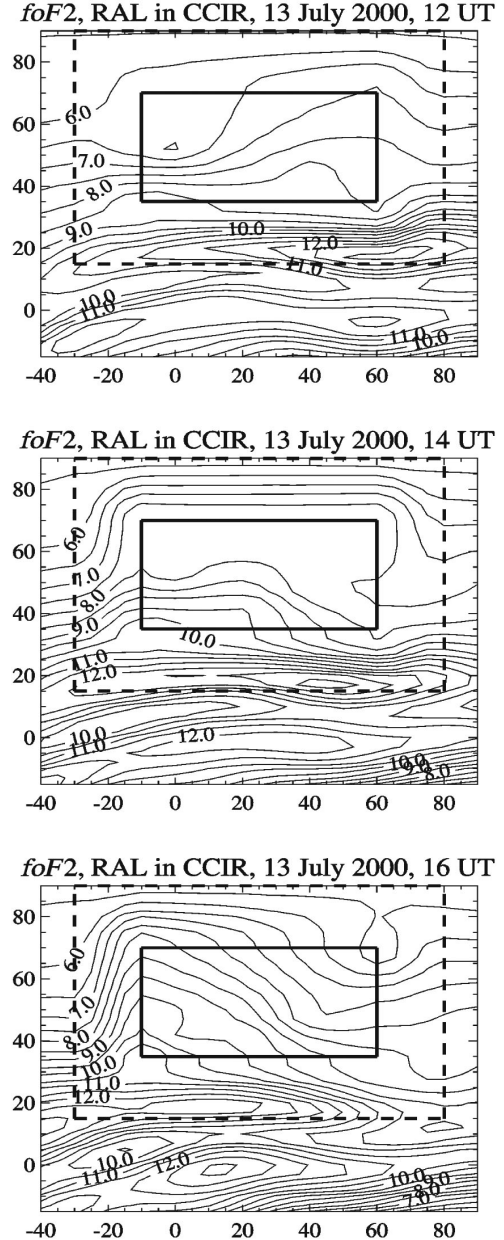
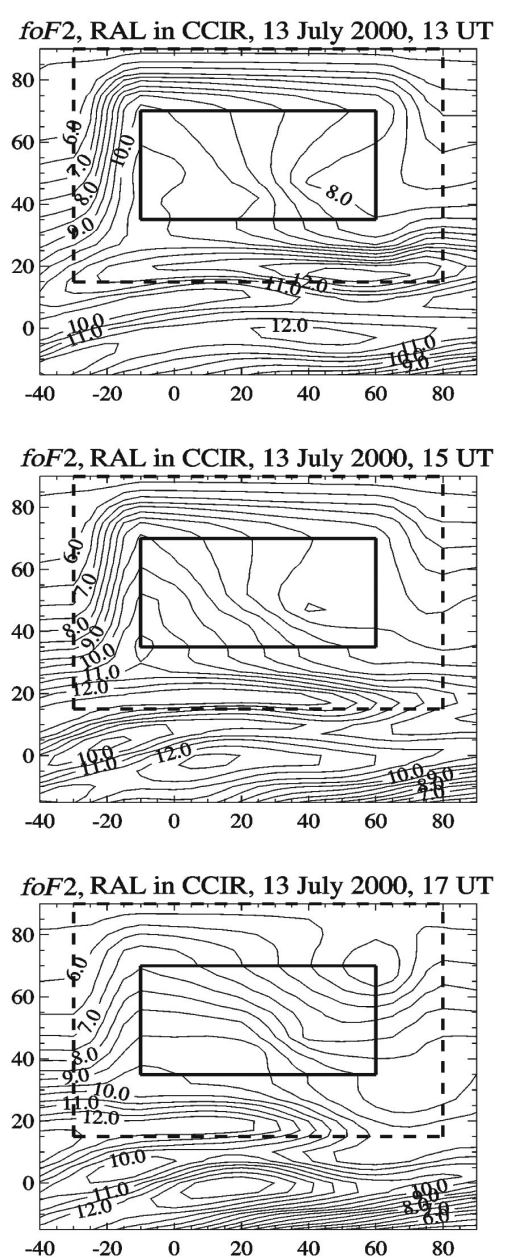

Fig. 6.12. EGNOS data grid example: «European» part of the hourly foF 2 grid for July 13, 1989,12 to $17 \mathrm{~h}$ UT. Contours of $f o F 2$ in $\mathrm{MHz}$ on a geographic map. Background: grid from the ITU-R (CCIR) monthly median map. The rectangular «RAL» region $\left(35^{\circ} \ldots 70^{\circ} \mathrm{N}, 10^{\circ} \mathrm{W} \ldots 60^{\circ} \mathrm{E}\right)$ is surrounded by a «buffer zone» for a smooth transition from background to «observed» values. The «RAL» grids have been produced from ionosonde scaling by means of an «instantaneous mapping» (nowcasting) method (L. Cander, 2002, pers. comm.). 
dustrial secrets) have been tested extensively, in the case of EGNOS by means of test data («storm scenarios») calculated by means of a modified and adapted version of the NeQuick «grid» model for 10 complete GPS days as well as by means of real data gained during the so-called testbed operation. The artificial («forward model») data are $1 \mathrm{~s}$ interval satellite to ground electron contents from 51 satellites (24 GPS, 24 GLONASS and 3 geostationary satellites) to 51 ground locations (34 «base stations» and 17 «users»). The model is based on «data grids» for the $F 2$ peak and for the topside of the ionosphere (Leitinger et al., 2001, 2002, 2003). The «data grids» are files with values over a regular grid in geographic coordinates (spacing $2.5^{\circ}$ in latitude and $5^{\circ}$ in longitude). For Europe the $F 2$ peak conditions were derived from ionosonde observations (fig. 6.12), but artificial positive storm effects were added for the American East Coast region. Figure 6.13 shows an example for slant electron content calculated for one of the 2601 satellite to receiving station links calculated for EGNOS Scenario 1 (Leitinger et al., 2002).
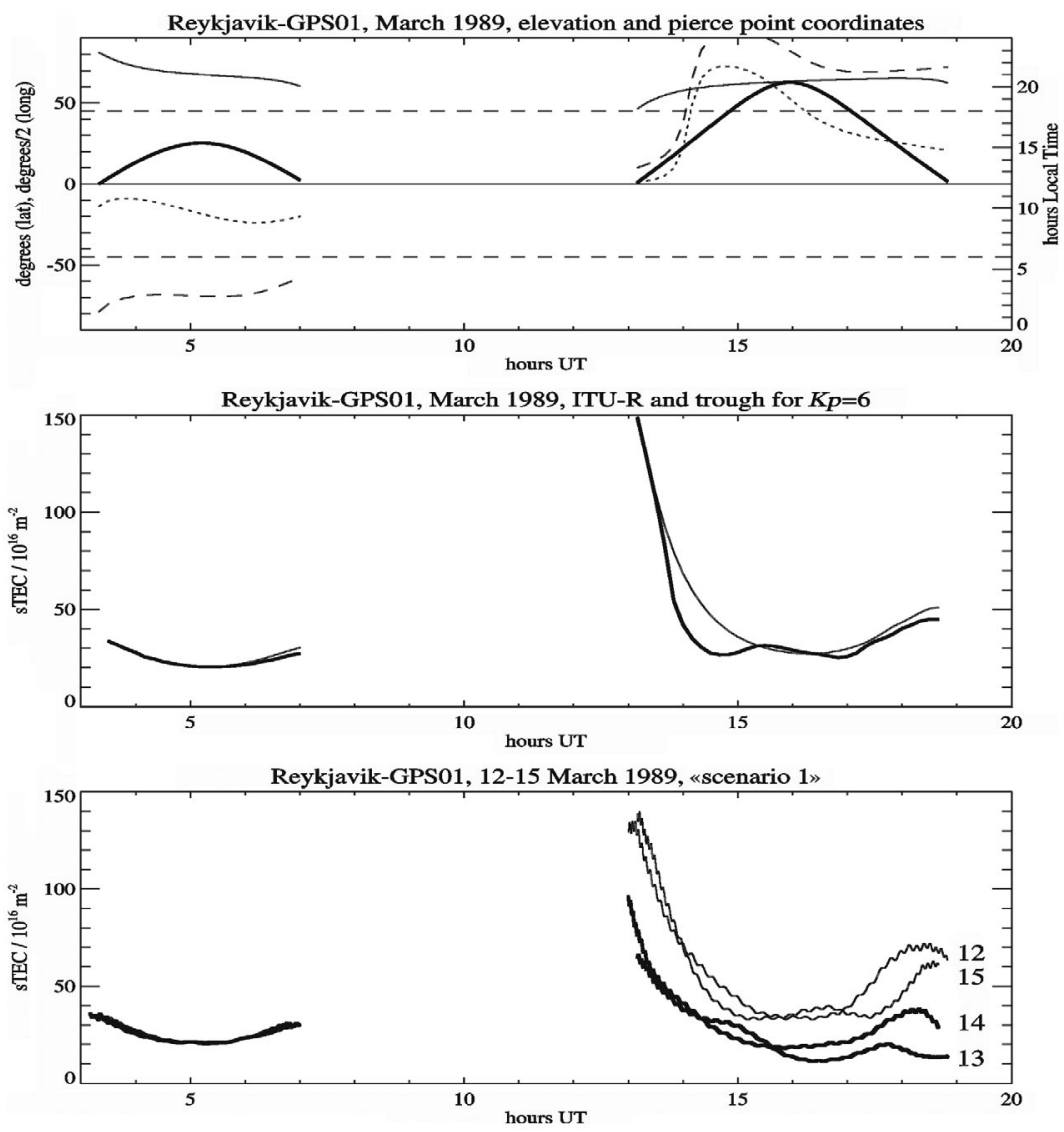

Fig. 6.13. Example from EGNOS «scenarios», receiving (RIMS) station Reykjavik $\left(64.1^{\circ} \mathrm{N}, 21.6^{\circ} \mathrm{W}\right)$, GPS satellite PRN 01, 12 to 15 March, 1989 («scenario» 1). Top: geometric situation (for 12 March). Satellite elevation (solid bold), pierce points (400 km) latitude (solid), longitude (dotted) and local time (dashed). Middle: March 198910 min slant electron content from NeQuick in ITU-R «map» mode and with trough modulation (bold). Bottom: «scenario» sTEC as delivered (spline interpolation from 10 min to $1 \mathrm{~s}$ data, TID modulation). 
Figure 6.13 shows an example for slant electron content calculated for one of the 2601 satellite to receiving station links calculated for EGNOS Scenario 1 (Leitinger et al., 2002).

The situation is substantially different for lower latitudes. The fixed pierce point height of $350 \mathrm{~km}$ is much too low for the equatorial anomaly region when the anomaly is «on» and the anomaly itself is not a very large scale «background» structure but a difficult «intermediate scale» one.

Furthermore, it is highly dynamic both in space and in time. Probably a change in concept will be needed for an extension of EGNOS to lower latitudes.

\subsection{Summary AND CONCLUSIONS}

It has been shown that the total electron content of the ionosphere can be determined with an accuracy of a few TECU which is sufficient to monitor large scale structures of the horizontal plasma distribution at least on a regional basis. On the one hand this monitoring contributes to understand the physics of the generation and development of ionospheric storms. On the other hand the TEC maps can be used to compensate or at least reduce ionosphere induced propagation errors.

Space based technical and science systems such as satellite communication, GNSS or satellite altimetry using trans-ionospheric radio waves with frequencies below $10 \mathrm{GHz}$, are principally affected by ionosphere induced propagation errors.

Fortunately, the GNSS technique itself provides a unique opportunity to monitor ionospheric key parameters continuously on regional and/or global scale in near-real-time.

A statistical estimation of horizontal TEC gradients reveals large scale gradients of up to about 6 TECU/1000 km under high solar activity conditions at an occurrence probability level of about $1 \%$.

Occasionally, during severe ionospheric storms this value may increase by a factor of 10 or even more.

Considering complex space weather events such as the event from 6 April 2000, it becomes clear that the ionosphere is modified in a complex and up to now unpredictable manner.

The close correlation of horizontal TEC gradients with geomagnetic activity indices gives the hope that TEC gradients my be forecasted by predicted geomagnetic indices. Large scale gradients degrade the performance of large area augmented systems for GNSS like WAAS and EGNOS.

If GNSS users are provided with ionospheric information, they can save higher accuracy and/or reliability of the measurements. So permanent monitoring of the ionosphere and the subsequent operational derivation of relevant information concerning the horizontal structure of the ionosphere and its dynamics is helpful for users in navigation, positioning and surveying.

\section{ACKNOWLEDGEMENTS}

The work reported here was part of the COST 271/WG 2 activities. Thus the authors are grateful to the COST 271 members for numerous help by coordinated work during campaigns, valuable suggestions and critical comments. We thank GPS data provider such as IGS, LOX and national agencies for getting free access to the GPS measurements used in our studies.

\section{REFERENCES}

BELEHAKI, A., N. JAKOWSKI and B.W. REINISCH (2003): Comparison of ionospheric ionisation measurements over Athens using ground ionosonde and GPS-derived TEC values, Radio Sci., 38 (6), 1105, 10.1029/2003RS002868.

Ciraolo, L., P. Spalla and P. Beni (1994): An analysis of consistency of TEC evaluated using pseudo 
range GPS observations, in Proceedings BSS94, edited by L. KERSLEY (University of Wales, Aberystwyth), 21-24.

DAVIES, K. and G.K. HaRTMAnN (1997): Studying the ionosphere with the global positioning system, Radio Sci., 84, 1695-1703.

Feltens, J., N. JAKOwSKI and C. Noll (2001): High-Rate SolarMax IGS/GPS Campaign «HIRAC/ SolarMax», CDDIS Bull., 16 (3).

FÖRSTER, M. and N. JAKOWSKI (2000): Geomagnetic storm effects on the topside ionosphere and plasmasphere: a compact tutorial and new results, Surv. Geophys., 21 (1), 47-87.

Foster, J.C., J.M. Holt, R.G. Musgrove and D.S. EvAnS (1986): Ionospheric convection associated with discrete levels of particle precipitation, Geophys. Res. Lett., 13, 656-659.

Harris, I.L., A.J. Mannucci, B.A. IiJima, U.J. Lindqwister, D. Muna, X. Pi and B.D. Wilson (2001): Ionospheric specification algorithms for precise GPS-based aircraft navigation, Radio Sci., 36, 287-298.

Hernandez-Pajares, M., J.M. Juan and J. SANZ (1997): Neural network modelling of the ionospheric electron content at global scale using GPS data, Radio Sci., 32, 1081-1089.

Hernandez-Pajares, M., J.M. JuAn and J. SAnZ (1999): New approaches in global ionospheric determination using ground GPS data, J. Atmos. Sol.-Terr. Phys., 61, 1237-1247.

Ho, M.C., A.J. Mannucci, U.J. Lindquister, X. Pi and T.T. Tsurutani (1996): Global ionosphere perturbations monitored by the worldwide GPS network, Geophys. Res. Lett., 23, 3219-3222.

Hochegger, G., B. NAVA, S.M. RADicella and R. Leitinger (2000): A family of ionospheric models for different uses, Phys. Chem. Earth (C), 25 (4), 307-310.

JAKOWSKI, N. (1996): TEC monitoring by using satellite positioning systems, in Modern Ionospheric Science, edited by H. KoHL, R. RÜSTER and K. SchlEGEL (EGS, Katlenburg-Lindau, ProduServ GmbH Verlagsservice, Berlin), 371-390.

JAKOWSKI, N. (1998): Generation of TEC maps over the COST area based on GPS measurements, in Proceedings of the 2nd COST 251 Workshop, 30-31 March 1998, Side, edited by A. VERNON, 51-57.

JaKowski, N. (2003): Plasma effects on GNSS applications, in Proceedings of the 3rd COST 271 Workshop, 23-27 September 2003, Spetses, Greece (on line: http://www.cost271.rl.ac.uk/).

JAKOWSKI, N. and E. SARDÓN (1996): Comparison of GPS/IGS-derived TEC data with parameters measured by independent ionospheric probing techniques, in Proceedings of the IGS, 19-21 March 1996, Silver Spring, 221-230.

JAKOWSKI, N., S. SCHLÜTER and E. SARDON (1999a): Total electron content of the ionosphere during the geomagnetic storm on January 10, 1997, J. Atmos. Sol.-Terr. Phys., 61, 299-307.

JAKOWSKI, N., K. HocKe, S. SCHLÜTER and S. HeISE (1999b): Space weather effects detected by GPS based TEC monitoring, in Proceedings of the Workshop on Space Weather, ESTEC, Noordwijk, WPP-155, 241-244.

JAKOWSki N., S. Heise, A. WeHRENPFenNIG and S. SCHLÜTER (2001): TEC monitoring by GPS - A possible contribution to space weather monitoring, Phys. Chem. Earth $(C), \mathbf{2 6}, 609-613$.

JAKOWSKI, N., A. WEHRENPFENNIG and S. HEISE (2002a): Ionospheric space weather effects monitored by GNSS signals, in Proceedings of the Ionospheric Effects Symposium, Alexandria, Virginia, edited by J.M. GoODMAN, 11-18.

JAKowski, N., A. Wehrenpfennig, S. Heise and I. Kutiev (2002b): Space weather effects on transionospheric radio wave propagation on 6 April 2000, in Proceedings of the 1st COST 271 Workshop, 24-29 September 2001, Sopron, Acta Geod. Geophys. Hung., 37 (2/3), 213-220.

JAKowski, N., S.M. StAnKov, D. KLAEHN and J. RuefFer (2003): Current developments in SWIPPA «Space Weather Impact on Precise Positioning Applications of GNSS», in Proceedings of the European Space Agency Workshop on Space Weather, ESTEC, 3-5 November 2003, Noordwijk, The Netherlands (on line: http://www.estec.esa.nl/wmwww/wma/spweather/workshops/spw_w5/proceedings.html).

Kersley, L., D. Malan, S.E. Pryse, Lu.R. Cander, R.A. Bamford, A. Belehaki, R. Leitinger, S.M. 
RADicella, C.N. Mitchell and P.S.J. SPENCER (2004): Total electron content - A key parameter in propagation: measurement and use in ionospheric imaging, Ann. Geophysics, 47 (suppl. to no. 2/3), 1067-1091 (this volume).

KLOBUCHAR, J.A. (1987): Ionospheric time-delay algorithm for single-frequency GPS users, IEEE Trans. Aerospace and Electr. Syst., AES-23, 325-331.

LEITINGER, R. (1990): The role of the atmosphere in satellite geodesy, radio stronomy and other applications of transatmospheric propagation of radio waves, in Proceedings of the I Beacon Satellite Symposium, San Miguel de Tucumán, Argentina, edited by J.R. Manzano.

LeITINGER, R. (1991): Ionospheric propagation errors, in Theoretical Problems in Space and Fusion Plasmas, edited by H.K. BIERnAT, S.J. BAUER and M. HeINDLER (ÖAW Wien), 147-156.

LeItinger, R. and E. Putz (1988): Ionospheric refraction errors and observables, in Atmospheric Effects on Geodetic Space Measurements (School of Surveying, University of New South Wales, Australia), edited by F.K. BrunNer, Monograph 12, 81-102.

Leitinger, R., B. NAVA, G. HochegGer and S.M. Radicella (2001): Ionospheric profilers using data grids, Phys. Chem. Earth (C), 26, 293-301.

LEITINGER, R., S.M. RADICELlA and B. NAVA (2002): Electron density models for assessment studies - new developments, Acta Geod. Geophys. Hung., 37, 183-193.

LEITINGER, R., S.M. RAdicElla and E. FeichteR (2003): The data grid method to actualize empirical electron density models, in Proceedings of the 2002 Ionospheric Effects Symposium, Alexandria, Virginia, edited by J.M. GOODMAN, 139-146.

SARdón, E., A. Rius and N. ZarRaOA (1994): Estimation of the receiver differential biases and the ionospheric total electron content from Global Positioning System observations, Radio Sci., 29, 577-586.

STAMPER, R., J. LILENSTEN and N. JAKOWSKI (2004): Nowcasting, forecasting and warning for ionospheric propagation: supporting databases, Ann. Geophysics, 47 (suppl. to no. 2/3), 945-955 (this volume).

Tsedilina, E.E., O.V. Weitsman and H. Soicher (1994): Time delay of transionospheric radio signals in a horizontally inhomogeneous ionosphere, Radio Sci., 29, 625-630.

Wilson, B.D. and A.J. MANnUCCI (1993): Instrumental biases in ionospheric measurements derived from GPS data, Proc. Inst. Navig. GPS, 93 (2), 1343-1351. 